\title{
Assembly of Protocell-like Vesicles in Micro-scale Hydrothermal Pores via Chaotic Thermal Convection
}

\author{
VIJAY RAVISANKAR, YASSIN A HASSAN AND VICTOR \\ M UGAZ
}

Texas A\&M University

Presenting Author: vijayravisankar@tamu.edu

A key unanswered question in the origin of life involves identifying physical mechanisms that drove the spontaneous assembly of lipid precursors into protocells under prebiotic conditions. Micro-scale porous environments that permeate mineral formation near alkaline hydrothermal vents provide a favorable chemical and physical environment, thus making them potential hotspots for these processes. Previous studies have shown aggregation of precursor molecules in thin cracks (diameter $\leq 100 \mu \mathrm{m})$ and under extreme thermal gradients $(100$ $1000{ }^{\circ} \mathrm{C} / \mathrm{mm}$ ) with infinite precursors. [1-2] However, image analysis of a cross-section of material retrieved from the Lost City vent reflects a high volume fraction of broader pores (diameter $\sim \mathrm{mm}$ to $\mathrm{cm}$ ). Recent studies in these broader pores have shown that chaotic convective flow naturally occurring due to modest thermal gradients $\left(1-5{ }^{\circ} \mathrm{C} / \mathrm{mm}\right)$ in these microenvironments exhibit enhanced bulk mixing and transport of chemical species. [3] Here we show that these chaotic convective flows can drive the assembly of vesicles formed by phosphatidylcholine, a representative phospholipid membrane constituent.

We performed computational simulations of thermally-driven convective flows and experimentally measured the size distribution of vesicles produced after $24 \mathrm{~h}$ of incubation using nanoparticle tracking analysis. Results obtained across an ensemble of pore-mimicking systems indicate that the growth of small unilamellar vesicles $(\sim 0.2 \mu \mathrm{m})$ into vesicles of size $1 \mu \mathrm{m}$ or larger is favored within the chaotic flow regime, as defined by the Lyapunov exponent Poincaré plots. We also used the Qcriterion to quantify the strength of the vortex structures present under conditions that produced the largest vesicles. This analysis reveals that localized vortex recirculation accompanied by enhanced bulk mixing via chaotic convection act synergistically to promote the assembly of vesicles in the protocell size range. These insights lay a foundation to link characteristics of the flow field (velocity, localized vortices, extent of chaos) within a pore network to the size distribution of vesicles produced, ultimately making it possible to identify conditions that favor protocell formation in hydrothermal systems.

[1] Budin, et al. (2009) JACS 131,9628-9629

[2] Mast, et al. (2013) PNAS 110,8030-8035.

[3] Priye, et al. (2017) PNAS 114,1275-1280. 